\title{
The Pregnancy-Unique Quantification of Emesis and Nausea (PUQE-24): Configural, measurement, and structural invariance between nulliparas and multiparas and across two measurement time points
}

\author{
Ayako Hada \\ Kitamura Institute of Mental Health Tokyo \\ Mariko Minatani \\ Life Value Creation Unit, NTT DATA Institute of Management Consulting, Inc., \\ Yukiko Yamagishi \\ Kitamura Institute of Mental Health Tokyo \\ Mikiyo Wakamatsu \\ Kagoshima University Faculty of Medicine School of Health Sciences \\ Gideon Koren \\ Ariel University \\ Toshinori Kitamura ( $\square$ kitamura@institute-of-mental-health.jp ) \\ Kitamura Institute of Mental Health Tokyo
}

Research Article

Keywords: PUQE-24, factor structure, measurement and structural invariance, parity, two observation occasions

Posted Date: March 2nd, 2021

DOI: https://doi.org/10.21203/rs.3.rs-238447/v1

License: (a) (1) This work is licensed under a Creative Commons Attribution 4.0 International License. Read Full License

Version of Record: A version of this preprint was published at Healthcare on November 15th, 2021. See the published version at https://doi.org/10.3390/healthcare9111553. 


\section{Abstract \\ Background}

The severity of nausea and vomiting of pregnancy (NVP) correlates with pregnancy complications. This study aimed to confirm the measurement and structural invariance of the 24-hour Pregnancy-Unique Quantification of Emesis and Nausea (PUQE-24) regarding parity and observation time among pregnant women during the first trimester.

\section{Methods}

Questionnaires including the PUQE-24 and the Health-Related Quality of Life for Nausea and Vomiting during Pregnancy (NVP-QOL) questionnaire were distributed to pregnant women from 10 to 13 weeks of gestation who were attending antenatal clinics. There were 383 respondents, and of these, 130 responded to the PUQE-24 again one week later.

\section{Results}

Confirmatory factor analysis of this single factor model showed good fit with the data: CFI $=1.000$. The PUQE-24 factor and NVPQOL factor were strongly correlated $(r=82)$. Configural, measurement, and structural invariance of the factor structure of the PUQE items were confirmed between primiparas and multiparas as well as at the test and retest observation occasions.

\section{Conclusion}

The findings suggested that using the PUQE-24 among pregnant women in the first trimester was robust in its factor structure. The PUQE-24 may be a promising tool as an easy and robust measure of the severity of nausea and vomiting among pregnant women.

\section{Background}

Nausea and vomiting of pregnancy (NVP) is a common health issue among pregnant women. NVP involves any degree or duration of nausea with or without vomiting or retching and which is not associated with other known causal factors. Almost 70\% of women worldwide experience NVP, with higher rates reported in East Asian countries [1]. Medical treatment is needed in severe cases. Nutritional disturbances, weight loss, dehydration, and ketonuria may lead to hospitalisation [2]. Hyperemesis gravidarum (HG) is considered the most severe form of NVP. Einarson et al. [1] reported that the prevalence of HG was $1.1 \%$. In addition to the emergence of somatic symptoms, a woman's quality of life (QOL) and ability to function are also impaired among those who suffered from severe NVP [3, 4]. A significant correlation between NVP and pregnancy complications was also reported. Women with NVP also have significant correlation with high blood pressure having had no prior history and preeclampsia [5]. Women with HG have a risk of having a low birth weight infant, an infant small for gestational age, or a preterm delivery [6, 7]. Women with $\mathrm{HG}$ also exhibited depression, post-traumatic stress disorder, and anxiety disorders $[8,9]$. For clinical and research use, a reliable and valid, as well as simple, measure for quantification of NVP severity is required.

The Pregnancy-Unique Quantification of Emesis and Nausea (PUQE) [10] is a severity measure used in studies to determine the burden or treatment outcome of NVP. The PUQE is a scoring system for nausea and vomiting during pregnancy, which consists of three items. The PUQE was developed for pregnant women on the basis of the Rhodes Index of Nausea and Vomiting (INV) [11] and focuses on three symptoms: nausea, vomiting, and retching. The original PUQE entailed rating the daily number of vomiting episodes, the length of nausea in hours per day, and the number of retching episodes per 12 hours. Its validation was confirmed by Koren et al [12]. To capture more comprehensive NVP severity, the PUQE was modified by Lacasse et al [13]. The Modified-PUQE (PUQE-24) is a scoring system per 24 hours with the same scoring calculation and interpretation as for the original PUQE. The PUQE is widely used as a scoring system to assess NVP severity in many countries $[14,15,16,17]$. Its use as the validated tool should be applied more frequently in better defining the severe end of HG [18].

The present study shows the psychometric properties of the PUQE-24 among pregnant women including confirmatory factor analysis (CFA) and configural, measurement, and structural invariance of the factor structure. We focused on the invariance of the 
factor structure between nulliparas and multiparas and between the test and retest occasions. When a psychological instrument is used in different populations or used at more than one measurement occasion, both selection of the best fit model of factor structure and confirmation of the postulation that the psychological instrument in question measures the same phenomena is needed. If this confirmation is not achieved, the instrument does not reflect the same phenomenon and the results may be biased. Invariance tests take several steps [19]. First, each group (e.g., nulliparas vs. multiparas) has the same pattern of the indicators and factors (configural invariance). Second, factor loadings for like indicators are invariant across groups (metric invariance; also known as weak factorial invariance). Third, intercepts of like items are invariant across groups (scalar invariance; also known as strong factorial invariance). Fourth, residuals (errors) of like items are invariant across groups (residual invariance; also known as strict factorial invariance). Fifth, variances of like factors are invariant across groups (factor variance invariance). Sixth, means of factors are invariant across groups (factor mean invariance). The second to fourth steps are called measurement invariance. The fifth and sixth steps are called structural invariance [19]. If one step is rejected, the next steps cannot be performed. We conducted tests for our hypotheses using this algorithm.

On the procedure of the data analysis and explanation, we followed the Consensus-based Standards for the selection of health Measurement Instruments (COSMIN) Study Design checklist [20]. It is recommended for designing studies and evaluating measurement properties, including content validity, structural validity, internal consistency, cross-cultural validity/measurement invariance, reliability, measurement error, criterion validity, hypotheses testing for construct validity, and responsiveness [21, 22]. The PUQE-24 has been evaluated its content validity $[10,12,13,23]$, hypothesis testing for construct validity $[13,24,25]$ and criterion validity [12]. To the best our knowledge, its validity including the measurement invariance has not been examined.

\section{Methods}

\section{Study procedures and participants}

This study was a longitudinal follow-up conducted at a 1-week interval because of the goal to examine measurement invariance between the test and retest occasions. We solicited approximately 1,500 pregnant women at 10 to 13 weeks of gestation at the antenatal clinic of two general hospitals and four private clinics located in Tokyo, Chiba, Ibaraki, and Kagoshima Prefectures in Japan. There were 383 total respondents (approximately $25 \%$ of those solicited). They were provided with a set of test and retest questionnaires and were asked to return the retest questionnaire 1 week later. Of the respondents, 130 sent back the retest questionnaire. Voluntary participation and anonymity were assured.

The participants gave written informed consent.

Responses for the two time occasions were matched by a predetermined number on the questionnaire. Pregnant women were excluded if they: (a) were not fluent in Japanese, (b) were aged under 20, (c) had eating disorders, (d) had symptoms of vaginal bleeding or abdominal pain, (e) had a subchorionic haematoma, or (f) experienced recurrent miscarriages.

The mean (SD) age of the participants was 31.9 (4.9) years old and the mean (SD) age of their partners was 33.5 (5.5) years old. Most of them were engaged in a relationship (94\%). Of the respondents, $43.9 \%$ were nulliparas and $54.8 \%$ were multiparas. This was a convenience sample. However, the sample consisted of those women receiving different types of obstetrical services in Japan. Data collection was conducted from January 2017 to May 2019.

\section{Measurements}

The 24-hour Pregnancy-Unique Quantification of Emesis and Nausea (PUQE-24) [23] is a self-measure rating (a) nausea (the length of nausea in hours for the last 24 hours), (b) vomiting (number of vomiting episodes in the last 24 hours), and (c) retching (the number of retching episodes in the last 24 hours) each with a 5-point scale. Higher scores indicate more severe NVP. The PUQE-24 was translated into Japanese by M.M. and T.K.

with permission from the original authors.

The bilingual author of the original version back-translated it and compared it with the original English.

The Japanese version [26] of the Health-Related Quality of Life for Nausea and Vomiting during Pregnancy questionnaire (NVP-QOL) [27] was used simultaneously as a measure of concurrent validity. This has 30 items with a 7-point scale measuring NVP and related QOL in the previous week. Higher scores indicate more severe NVP and worse QOL. The NVP-QOL has a single factor structure [26]. 


\section{Data analysis}

The PUQE-24 should have a single-factor structure because it consists of only three items. Hence, we only performed exploratory factor analyses (EFAs) for a single-factor structure. After calculating mean, SD, skewness, and kurtosis of each PUQE-24 item, we examined a factorability check of the PUQE-24 with the Keiser-Meyer-Olkin (KMO) index and Bartlett's sphericity test [28]. A singlefactor EFA derived the factor loading of the PUQE-24 items. Then, a CFA of the single-factor analysis was examined and checked for its goodness-of-fit. The fit of the models was checked in terms of chi-squared and comparative fit index (CFI). A good fit would be indicated by $\chi^{2} / d f<2$, and CFI $>.97$, and an acceptable fit by $\chi^{2} / d f<3$, and CFI $>.95[29,30]$.

The model's configural, measurement, and structural invariance was examined across parity and observation occasions. Starting from the configural invariance, we went through metric, scalar, residual, and factor variance invariances to factor mean invariances $[19,31]$. The progress from one step to the next was judged as 'accepted' if (a) the $\chi^{2}$ decrease was not significant for the $d f$ difference, (b) the decrease of CFI was less than .01, or (c) the increase of root mean square of error approximation (RMSEA) was less than $0.01[32,33]$. We applied this procedure because a $\chi^{2}$ decrease is strongly sensitive to the sample size $(M)$ and, particularly in the case of a large sample, produces an unreasonable rejection of invariance.

\section{Results}

Mean, SD, skewness, and kurtosis of the three PUQE-24 items are in Table 1. One item showed a slightly high skewness (2.93) and kurtosis (9.68). The KMO was .625 and Bartlett's sphericity was 190.796 (3) $(p<.001)$. Therefore, the data appeared factorable. Factor loading of each item in the single-factor model is in Table 1 . This model explained $61 \%$ of the whole variance. Confirmatory factor analysis of this single factor model showed good fit with the data: CFI $=1.000$. The PUQE-24 factor and NVP-QOL factor was strongly correlated $(r=.82)$.

Table 1. Mean, SD, skewness, and kurtosis of PUQE-24 items $(N=378)$

$$
\text { ITEM }
$$

Mean SD Skewness Kurtosis Factor loading of 1-

factor model

\begin{tabular}{lllllllc}
\hline 1 & $\begin{array}{l}\text { In the last } 24 \text { hours, how long have you felt nauseated or sick } \\
\text { to your stomach? }\end{array}$ & 377 & 3.1 & 1.5 & 0.00 & $\square 1.31$ & .77 \\
2 & 378 & 1.3 & 0.6 & 2.93 & 9.68 & .46 \\
\hline In the last 24 hours, have you vomited or thrown up? & $\begin{array}{l}\text { In the last } 24 \text { hours, how many times have you had retching } \\
\text { or dry heaves without bringing anything up? }\end{array}$ & 378 & 2.1 & 1.3 & 1.00 & $\square 0.02$ & .70 \\
\hline
\end{tabular}

Configural and measurement invariances are accepted between primiparas and multiparas as well as between the test and retest occasions (Table 2). Factor mean also did not differ between primiparas and multiparas as well as between the test and retest occasions (Table 3).

Table 2. Measurement and structural invariance of the PUQE-24

\begin{tabular}{|c|c|c|c|c|c|c|c|c|c|}
\hline & $\chi^{2}$ & $d f$ & $\chi^{2} / d f$ & $\Delta \chi^{2}(d f)$ & CFI & $\triangle \mathrm{CFI}$ & RMSEA & $\triangle \mathrm{RMSEA}$ & Judgement \\
\hline \multicolumn{10}{|c|}{ Nulliparas $(n=168)$ vs. multiparas $(n=210)$} \\
\hline Configural & 0.000 & 0 & 0 & Ref & 1.000 & Ref & 0.000 & Ref & ACCEPT \\
\hline Metric & 0.949 & 2 & 0.474 & $0.949(2) * *$ & 1.000 & 0.000 & 0.000 & 0.000 & ACCEPT \\
\hline Scalar & 3.788 & 5 & 0.758 & $2.840(3) *$ & 1.000 & 0.000 & 0.000 & 0.000 & ACCEPT \\
\hline Residual & 4.325 & 8 & 0.541 & 0.540 (3) NS & 1.000 & 0.000 & 0.000 & 0.000 & ACCEPT \\
\hline Factor variance & 4.356 & 9 & 0.484 & 0.027 (1) NS & 1.000 & 0.000 & 0.000 & 0.000 & ACCEPT \\
\hline \multicolumn{10}{|c|}{ Time $1(n=383)$ vs. Time $2(n=130)$} \\
\hline Configural & 0.000 & 0 & 0 & Ref & 1.000 & Ref & 0.000 & Ref & ACCEPT \\
\hline Metric & 1.089 & 2 & 0.545 & 1.089 (2) NS & 1.000 & 0.000 & 0.000 & 0.000 & ACCEPT \\
\hline Scalar & 2.940 & 5 & 0.588 & 1.851 (3) NS & 1.000 & 0.000 & 0.000 & 0.000 & ACCEPT \\
\hline Residual & 9.211 & 8 & 1.151 & $6.271(3) \mathrm{NS}$ & 0.995 & 0.005 & 0.017 & 0.017 & ACCEPT \\
\hline Factor variance & 10.367 & 9 & 1.152 & $1.089(2) \mathrm{NS}$ & 0.994 & 0.001 & 0.017 & 0.000 & ACCEPT \\
\hline
\end{tabular}

$* p<.05 ;{ }^{* *} p<.01 ; * * * p<.001$; NS, not significant. 
Table 3 Factior mean invariance of the PUQE-24

\begin{tabular}{lc}
\hline & Differences in factor mean (SE) \\
\hline Multiparas as compared with nulliparas & $0.148(0.103) \mathrm{NS}$ \\
Time 2 as compared with Time 1 & $\square 0.136(0.105) \mathrm{NS}$ \\
\hline
\end{tabular}

$* p<.05 ; * * p<.01 ; * * * p<.001$; NS, not significant; SE, standard error.

\section{Discussion}

The present study showed that the single-factor structure of the PUQE-24 was robust among pregnant Japanese women. Its structure was invariant regardless of parity as well as observation times. Concurrent validity with the NVP-QOL scores was also excellent. According to Ebrahimi et al. [23], the PUQE-24 has exactly reflected pregnant women's severity of symptoms of NVP during one day. Taking into account the PUQE-24's simplicity, we think that the use of the PUQE-24 in clinical and research settings in antenatal maternal care is extremely promising. This is particularly the case when clinicians and researchers wish to distinguish between cases of severe NVP, most likely due to HG, and mild and moderate cases. The PUQE-24 may be used as an outcome measure of intervention by midwives and other perinatal health professionals.

There are several limitations to this study. Our study sample size was medium and based on a convenience sample. One of the inclusion criteria was pregnant women at 10 to 13 weeks of gestation. Though we intended to have a homogeneous population of pregnant women for this study sample, different results may have been produced if women at different weeks of gestation had been studied. Yet, the range of $10-13$ weeks gestation is very slim and not likely to increase variability.

Hence, careful generalisation is needed.

The findings were based on self-reporting. Further examination of the degree of concordance between their reports and clinical observers' or family members' reports is needed.

Taking these drawbacks into consideration, the PUQE-24 appears to be a promising tool as an easy and robust measure of severity of NVP among pregnant women.

\section{Conclusion}

The findings suggested that using the PUQE-24 among pregnant women in the first trimester was robust in its factor structure. The PUQE-24 may be a promising tool as an easy and robust measure of the severity of nausea and vomiting among pregnant women.

\section{Abbreviations}

CFA: confirmatory factor analysis

CFI: comparative fit index

COSMIN: COnsensus-based Standards for the selection of health Measurement INstruments.

EFA: exploratory factor analyses

HG: Hyperemesis gravidarum

INV: Rhodes Index of Nausea and Vomiting

KMO: Keiser-Meyer-Olkin index

NVP: nausea and vomiting of pregnancy

NVP-QOL: Health-Related Quality of Life for Nausea and Vomiting during Pregnancy questionnaire

PUQE-24: 24-hour Pregnancy-Unique Quantification of Emesis and Nausea 
QOL: quality of life

RMSEA: root mean square of error approximation

SD: standard deviation

\section{Declarations}

\section{Acknowledgements}

We are grateful for all of the participants and the Japanese Red Cross Medical Centre, Endou Ladies Clinic, Kubonoya Women's Hospital, Tsuchiya Obsteric \& Gynaecology Clinic, Aiiku Hospital, and Nakae Obstetiric \& Gynaecology Clinic.

\section{Funding}

The authors declare that they have no funding or research grants received in the course of study.

\section{Availability of data and materials}

The datasets used and analyzed in the present study are available from the corresponding author upon reasonable request.

\section{Ethical approval and consent to participate}

This study was approved by the Institutional Review Board (IRB) of the Kitamura Institute of Mental Health Tokyo (No. 2015052301) and Kagoshima University (No.170247). All the participants gave written informed consent after understanding the study rationale and procedure. The authors assert that all procedures contributing to this study comply with the ethical standards of the National and Institutional Committees on human experimentation and with the Helsinki Declaration of 1975 as revised in 2008. All participants taking part in the study provided written informed consent.

\section{Consent to publication}

Not applicable

\section{Conflict interests}

The authors declare that they have no conflicts of interest.

\section{Author's contribution}

$M M$ and TK set up the research design. GK provided the instrument and commented the manuscript. $M M, A H, Y Y$ and $M W$ collected data. $\mathrm{AH}$ and TK analysed data. AH and TK wrote the manuscript.

\section{References}

1. Einarson TR, Piwko C, Koren G. Quantifying the global rates of nausea and vomiting of pregnancy: a meta analysis. J Popul Ther Clin Pharmacol = J la Ther des Popul la Pharmacol Clin. 2013;20(2):e171-83. http://europepmc.org/abstract/MED/23863575

2. Miller F. Nausea and vomiting in pregnancy: The problem of perception-is it really a disease? Am J Obstet Gynecol. 2002;186(5):S182-S183. doi:10.1067/mob.2002.122594

3. Attard CL, Kohli MA, Coleman S, et al. The burden of illness of severe nausea and vomiting of pregnancy in the United States. Am J Obstet Gynecol. 2002;186(5, Supplement 2):S220-S227. doi:https://doi.org/10.1067/mob.2002.122605

4. Heitmann K, Nordeng H, Havnen GC, Solheimsnes A, Holst L. The burden of nausea and vomiting during pregnancy: severe impacts on quality of life, daily life functioning and willingness to become pregnant again - results from a cross-sectional study. BMC Pregnancy Childbirth. 2017;17(1):75. doi:10.1186/s12884-017-1249-0 
5. Bustos M, Venkataramanan R, Caritis S. Nausea and vomiting of pregnancy - What's new? Auton Neurosci. 2017;202:62-72. doi:https://doi.org/10.1016/j.autneu.2016.05.002

6. Dodds L, Fell DB, Joseph KS, Allen VM, Butler B. Outcomes of Pregnancies Complicated by Hyperemesis Gravidarum. Obstet Gynecol. 2006;107(2).

https://journals.Iww.com/greenjournal/Fulltext/2006/02000/Outcomes_of_Pregnancies_Complicated_by_Hyperemesis.13.aspx.

7. Veenendaal MV, van Abeelen AF, Painter RC, van der Post JA, Roseboom TJ. Consequences of hyperemesis gravidarum for offspring: a systematic review and meta-analysis. Int J Obstet Gynaecol. 2011;118(11):1302-1313.

https://doi.org/10.1111/j.1471-0528.2011.03023.x

8. Grooten IJ, Roseboom TJ, Painter RC. Barriers and Challenges in Hyperemesis Gravidarum Research. Nutr Metab Insights. 2016;8(Suppl 1):33-39. https://doi.org/10.4137/NMI.S29523

9. McCarthy FP, Khashan AS, North RA, et al. A prospective cohort study investigating associations between hyperemesis gravidarum and cognitive, behavioural and emotional well-being in pregnancy. Plos One. 2011;6(11).

10. Koren G, Boskovic R, Hard M, Maltepe C, Navioz Y, Einarson A. Motherisk: PUQE (pregnancy-unique quantification of emesis and nausea) scoring systm for nausea and vomiting of pregnancy. Am J Obstet Gynecol.2002;186: s210-214.

11. Rhodes VA, Watson PM, Johnson MH. Development of reliable and valid measures of nausea and vomiting. Cancer Nurs. 1984;7:33-42.

12. Koren G, Piwko C, Ahn E, et al. Validation studies of the Pregnancy Unique-Quantification of Emesis (PUQE) scores. J Obstet Gynaecol. 2005;25:241-244.

13. Lacasse A, Rey E, Ferreira E, Morin C, Bérard A. Validity of a modified Pregnancy-Unique Quantification of Emesis and Nausea (PUQE) scoring index to assess severity of nausea and vomiting of pregnancy. Am J Obstet Gynecol. 2008;198(1):71-e1.

14. Birkeland E, Stokke G, Tangvik RJ, et al. Norwegian PUQE (Pregnancy-Unique Quantification of Emesis and Nausea) identifies patients with hyperemesis gravidarum and poor nutritional intake: a prospective cohort validation study. Plos One. 2015;10(4).

15. Choi HJ, Bae YJ, Choi JS, et al. Evaluation of nausea and vomiting in pregnancy using the Pregnancy-Unique Quantification of Emesis and Nausea scale in Korea. Obstet Gynecol Sci. 2018;61(1):30-37. https://doi.org/10.5468/ogs.2018.61.1.30

16. Dochez V, Dimet J, David-Gruselle A, Le Thuaut A, Ducarme G. Validation of specific questionnaires to assess nausea and vomiting of pregnancy in a French population. In J Gynaecol Obstet: the official organ of the International Federation of Gynaecology and Obstetrics. 2016;134(3):294-298. https://doi.org/10.1016/j.ijgo.2016.01.023

17. Isbir GG, Mete S. The effect of counselling on nausea and vomiting in pregnancy in Turkey. Sex Reprod Healthcare. 2016;7:3845. https://doi.org/10.1016/j.srhc.2015.11.005

18. Koren G, Cohen R. Measuring the severity of nausea and vomiting of pregnancy; a 20-year perspective on the use of the pregnancy-unique quantification of emesis (PUQE). J Obstet Gynaecol (Lahore) [Internet]. 2020 Aug 19;1-5. Available from: https://doi.org/10.1080/01443615.2020.1787968

19. Vandenberg RJ, Lance CE. A review and synthesis of the measurement invariance literature: Suggestions, practices, and recommendations for organizational research. Organ Res Methods. 2000;3(1):4-69. doi:10.1177/109442810031002 .

20. Mokkink LB, Prinsen CAC, Patrick DL, Alonso J, Bouter LM, De Vet HC, et al. COSMIN study design checklist for patient-reported outcome measurement instruments. Dep Epidemiol Biostat. 2019;

21. Mokkink LB, de Vet HCW, Prinsen CAC, Patrick DL, Alonso J, Bouter LM, et al. COSMIN Risk of Bias checklist for systematic reviews of patient-reported outcome measures. Qual Life Res [Internet]. 2018;27(5):1171-9. Available from:

https://doi.org/10.1007/s11136-017-1765-4

22. Prinsen CAC, Mokkink LB, Bouter LM, Alonso J, Patrick DL, de Vet HCW, et al. COSMIN guideline for systematic reviews of patient-reported outcome measures. Qual Life Res [Internet]. 2018;27(5):1147-57. Available from:

https://doi.org/10.1007/s11136-018-1798-3

23. Ebrahimi N, Maltepe C, Bournissen FG, Koren G. Nausea and Vomiting of Pregnancy: Using the 24-hour Pregnancy-Unique Quantification of Emesis (PUQE-24) Scale. J Obstet Gynaecol Canada. 2009;31:803-807. doi:10.1016/S1701-2163(16)34298-0

24. Koot, M. H., Grooten, I. J., van der Post, J. A., Bais, J. M., Ris-Stalpers, C., Leeflang,M. M., ... Kleiverda, G. (2020). Determinants of disease course and severity in hyperemesis gravidarum. European Journal of Obstetrics \& Gynecology and Reproductive Biology,245, 162-167. 
25. McParlin C, Carrick-Sen D, Steen IN, Robson SC. Hyperemesis in pregnancy study: A pilot randomised controlled trial of midwifeled outpatient care. Eur J Obstet Gynecol Reprod Biol [Internet]. 2016;200:6-10. Available from:

http://www.sciencedirect.com/science/article/pii/S0301211516300525

26. Yamada F, Minatani M, Yamagishi Y, Hada A. Kitamura T. Nausea and Vomiting of Pregnancy Specific Health Related Quality of Life Questionnaire: Factor structure and measurement invariance among Japanese pregnant women. 2020;(under review)

27. Magee LA, Chandra K, Mazzotta P, Stewart D, Koren G, Guyatt GH. (2002). Development of a health-related quality of life instrument for nausea and vomiting of pregnancy. Am J Obstet Gynecol. 2002;186(5):S232-S238.

28. Burton LJ, Mazerolle SM. Survey instrument validity Part I: Principles of survey instrument development and validity in athletic training education research. Athl Train Educ J. 2011;6:27-35.

29. Bentler PM. Comparative fit indexes in structural models. Psychol Bull. 1990;107(2):238.

30. Schermelleh-Engel K, Moosbrugger $\mathrm{H}$, Müller H. Evaluating the fit of structural equation models: Tests of significance and descriptive goodness-of-fit measures. Methods Psychol Res. 2003;8(2):23-74.

31. van de Vijver FJR, Leung K. Methodological issues in psychological research on culture. J Cross Cult Psychol. 2000;31(1):3351.

32. Chen FF. Sensitivity of goodness of fit indexes to lack of measurement invariance. Struct Equ Modeling. 2007;14(3):464-504.

33. Cheung GW, Rensvold RB. Evaluating goodness-of-fit indexes for testing measurement invariance. Struct Equ Modeling. 2002;9(2):233-255. 